\title{
Uniform gauge for D1-brane in general background
}

\section{Josef Kluson̆}

Department of Theoretical Physics and Astrophysics, Faculty of Science, Masaryk University, Kotlářská 2, 611 37, Brno, Czech Republic

E-mail: klu@physics.muni.cz

ABSTRACT: We construct uniform gauge D1-brane action in general background. We also discuss how this action transforms under double Wick rotation and determine transformation properties of background fields.

KEYwords: D-branes, AdS-CFT Correspondence

ARXiv EPrint: 1503.08025 


\section{Contents}

1 Introduction and summary $\quad 1$

2 Uniform gauge fixing for D1-brane in general background 3

3 Double Wick rotation $\quad 10$

\section{Introduction and summary}

It is well known that the central role in our understanding of the AdS/CFT correspondence is played by integrability. ${ }^{1}$ Explicitly, the scaling dimensions in planar $\mathcal{N}=4 \mathrm{SYM}$ can be described by the corresponding energy levels of a string on $\operatorname{AdS}_{5} \times S^{5}$ and these levels can be computed using the integrability of the string, where the spectrum of $\operatorname{AdS}_{5} \times S^{5}$ superstring is determined by means of the thermodynamic Bethe ansatz that is applied to a doubly Wick rotated version of world-sheet theory [7, 9, 10]. It is important to stress that this double Wick rotation is applied on the light cone gauge fixed $\mathrm{AdS}_{5} \times S^{5}$ string which is not Lorentz invariant. As a result the double Wick rotation leads to the non-equivalent quantum field theory known as a mirror theory. Given mirror theory was very useful for the description of planar scattering amplitudes [11-13]. Then we can ask the question whether mirror symmetry has deeper physical meaning rather than to be considered as a technical tool. In other words we would like to see whether the mirror theory arises by uniform gauge fixing of a free string in some background. This was shown firstly in [6] for the case of the bosonic string in $\mathrm{AdS}_{5} \times S^{5}$ background and its integrable deformation [14-18], for review, see [19]. The analysis was generalized to the Green-Schwarz superstring in [15] where it was also suggested how the dilaton and Ramond-Ramond fields should transform under mirror symmetry, even if it is not completely clear whether the mirror background is the string background as well.

It is well known that string theories contain another extended objects in their spectrum as for example D-branes [8]. D1-brane has the special meaning since it is two dimensional object that is very similar to the fundamental string. Further, D1-brane couples to the dilaton and Ramond-Ramond fields through its Dirac-Born-Infeld action and Wess-Zummino term. For that reason the study of D1-brane in more general background could be very useful for the analysis of the integrability of these general backgrounds. Previously we analyzed the integrability of D1-brane on group manifold [3] and we showed that this theory is integrable. Now we would like to extend the analysis of D1-branes further and try to find uniform light cone gauge fixed D1-brane in general background with the presence of the Ramond-Ramond fields and dilaton, where the uniform light cone gauge was introduced

\footnotetext{
${ }^{1}$ For review and extensive list of references, see $[1,2]$.
} 
for the string in $\mathrm{AdS}_{5} \times S^{5}$ background [21, 22]. Then we analyze how the gauge fixed D1-brane action transforms under double Wick rotation and how the background fields should transform in order to preserve the form of the gauge fixed action. More precisely, in order to find the gauge fixed form of the D1-brane action we firstly find the Hamiltonian formulation of D1-brane in general background. We identify three first class constraints where two of them correspond to the world-volume diffeomorphism invariance and the last one to the gauge invariance of given theory related to the presence of the gauge field $A_{\alpha}$ on the world-volume of D1-brane. We fix these first class constraints by imposing uniform light cone gauge and then we find the Hamiltonian for the physical degrees of freedom only. Finally we determine the Lagrangian for the physical degrees of freedom which is the main goal of this paper. This Lagrangian could be starting point for further analysis of more general integrable systems, as for example for the analysis of giant magnon solution in $\kappa$-deformed background, for the analysis of the world-sheet S-matrix with the presence of Ramond-Ramond fields and so on. However in given article we restrict ourselves to the question how gauge fixed D1-brane Lagrangian behaves under double Wick rotation. From the requirement that the double Wick rotated action should have the same form as the original one we determine mirror background. However due to the fact that the gauge fixed action possesses constant electric flux that is proportional to the number of fundamental strings we find that this flux is involved in the transformation properties of the background fields. Of course, this is a trivial situation in case of a constant dilaton and zero RamondRamond one form as in case of $\mathrm{AdS}_{5} \times S^{5}$ background since then we can rescale the spatial coordinate of D1-brane theory so that the dependence on the electric flux and constant dilaton appears as a constant prefactor in front of the action. In other words in case of the background with constant dilaton and zero Ramond-Ramond fields the action behaves under the double Wick rotation as uniform gauge fixed bosonic string. However there is an important difference that makes the meaning of the double Wick rotation in case of gauge fixed D1-brane theory unclear. It was argued in [6] that the double Wick rotation for the bosonic string in $\mathrm{AdS}_{5} \times S^{5}$ gives the mirror background with non-trivial dilaton. On the other hand the double Wick rotation in case of gauge fixed D1-brane action implies that $e^{-2 \Phi}+\left(C^{(0)}\right)^{2}$ does not transform and hence we would find that non-trivial dilaton in the mirror background should be supported corresponding non-trivial Ramond-Ramond zero form. In other words the double Wick rotation in case of gauge fixed D1-brane predicts different transformation of the background fields than in case of the double Wick rotation on the world sheet of gauge fixed fundamental string [6]. Further, when we apply double Wick rotation to the case of gauge fixed D1-brane in $\kappa$ deformed $\mathrm{AdS}_{3} \times S^{3}$ background we find that Wick rotated theory cannot be equivalent to the original one due to the presence of non-trivial Ramond-Ramond two form [16]. In other words the double Wick rotation in case of the gauge fixed D1-brane theory in general background cannot give the action that has formally the same form as the original one. It is possible that the origin of this discrepancy is in the fact that the double Wick rotation performed in case of the fundamental string corresponds to the T-duality in $\phi$ coordinates, then performing analytic continuing $t, \phi \rightarrow i \phi,-i t$ and doing another $T$-duality in the new $\phi$ coordinate [15]. On the other hand these transformations are more involved in case of D1-brane theory since during T-duality 
transformations the dimensions of D-brane change. Further, the standard treatment of T-duality transformations in case of D1-brane theory is performed when the static gauge is imposed, for review see [20]. However in case of the sequence of T-duality rules performed above the static gauge is imposed by requirement that the variable dual to $\phi$ coincides with spatial coordinate $\sigma$. For that reason it would be more appropriate to find how D1-brane transforms under T-duality transformations without imposing static gauge. We hope to return to this problem in future.

Let us outline our results. We derive the uniform gauge fixed D1-brane action in general background. This action could be very useful for the study of the integrability of this theory in $\kappa$-deformed background with non-trivial dilaton and Ramond-Ramond fields. It could be also very interesting to study giant magnon solution on given D1-brane. On the other hand the double Wick rotation in given theory is subtle and should be clarified further.

The structure of given paper is as follows. In the next section 2 we perform Hamiltonian formulation of D1-brane in general background. Then we perform uniform gauge fixing and determine Lagrangian density for the physical degrees of freedom. In section 3 we perform the double Wick rotation in given action and discuss conditions under which the double Wick rotated action has the same form as the original one.

\section{Uniform gauge fixing for D1-brane in general background}

In this section we find the uniform gauge fixed form of D1-brane in general background. We begin with the D1-brane action in general background that has the form

$$
\begin{aligned}
S= & -T_{D 1} \int d \tau d \sigma e^{-\Phi} \sqrt{-\operatorname{det}\left(g_{\alpha \beta}+b_{\alpha \beta}+\left(2 \pi \alpha^{\prime}\right) F_{\alpha \beta}\right)} \\
& +T_{D 1} \int d \tau d \sigma\left[C^{(0)}\left(b_{\tau \sigma}+\left(2 \pi \alpha^{\prime}\right) F_{\tau \sigma}\right)+C_{\tau \sigma}^{(2)}\right]
\end{aligned}
$$

where

$$
g_{\alpha \beta}=g_{M N} \partial_{\alpha} x^{M} \partial_{\beta} x^{N}, \quad b_{\alpha \beta}=b_{M N} \partial_{\alpha} x^{M} \partial_{\beta} x^{N}, \quad C_{\tau \sigma}^{(2)}=C_{M N}^{(2)} \partial_{\tau} x^{M} \partial_{\sigma} x^{N},
$$

and where $x^{M}(\tau, \sigma)$ are embedding coordinates for D1-brane in given background. Further, $F_{\alpha \beta}=\partial_{\alpha} A_{\beta}-\partial_{\beta} A_{\alpha}$ is the field strength of the world-volume gauge field $A_{\alpha}, \alpha=\tau, \sigma$. Finally $T_{D 1}$ is D1-brane tension $T_{D 1}=\frac{1}{2 \pi \alpha^{\prime}}$.

Before we proceed to the Hamiltonian formulation of the action (2.1) it is useful to use following formula

$$
\operatorname{det}\left(g_{\alpha \beta}+b_{\alpha \beta}+\left(2 \pi \alpha^{\prime}\right) F_{\alpha \beta}\right)=\operatorname{det} g_{\alpha \beta}+\left(b_{\tau \sigma}+\left(2 \pi \alpha^{\prime}\right) F_{\tau \sigma}\right)^{2}
$$


that holds in two dimensions only. Then from the action (2.1) we find momenta conjugate to $x^{M}, A_{\sigma}$ and $A_{\tau}$ respectively

$$
\begin{aligned}
p_{M}= & T_{D 1} \frac{e^{-\Phi}}{\sqrt{-\operatorname{det} g-\left(\left(2 \pi \alpha^{\prime}\right) F_{\tau \sigma}+b_{\tau \sigma}\right)^{2}}}\left(g_{M N} \partial_{\alpha} x^{N} g^{\alpha \tau} \operatorname{det} g+\left(\left(2 \pi \alpha^{\prime}\right) F_{\tau \sigma}+b_{\tau \sigma}\right) b_{M N} \partial_{\sigma} x^{N}\right) \\
& +T_{D 1}\left(C^{(0)} b_{M N} \partial_{\sigma} x^{N}+C_{M N}^{(2)} \partial_{\sigma} x^{N}\right), \\
\pi^{\sigma}= & \frac{e^{-\Phi} T_{D 1}\left(2 \pi \alpha^{\prime}\right)\left(\left(2 \pi \alpha^{\prime}\right) F_{\tau \sigma}+b_{\tau \sigma}\right)}{\sqrt{-\operatorname{det} g-\left(\left(2 \pi \alpha^{\prime}\right) F_{\tau \sigma}+b_{\tau \sigma}\right)^{2}}}+T_{D 1}\left(2 \pi \alpha^{\prime}\right) C^{(0)}, \quad \pi^{\tau} \approx 0 .
\end{aligned}
$$

Using these relations we find that the bare Hamiltonian is equal to

$$
H=\int d \sigma\left(p_{M} \partial_{\tau} x^{M}+\pi^{\sigma} \partial_{\tau} A_{\sigma}-\mathcal{L}\right)=\int d \sigma \pi^{\sigma} \partial_{\sigma} A_{\tau}
$$

while we have three primary constraints

$$
\begin{aligned}
& \mathcal{H}_{\sigma} \equiv p_{M} \partial_{\sigma} x^{M} \approx 0, \quad \pi^{\tau} \approx 0 \\
& \mathcal{H}_{\tau} \equiv \Pi_{M} g^{M N} \Pi_{N}+\left(T_{D 1}^{2} e^{-2 \Phi}+\left(\frac{\pi^{\sigma}}{\left(2 \pi \alpha^{\prime}\right)}-T_{D 1} C^{(0)}\right)^{2}\right) g_{M N} \partial_{\sigma} x^{M} \partial_{\sigma} x^{N}
\end{aligned}
$$

where

$$
\Pi_{M} \equiv p_{M}-\frac{\pi^{\sigma}}{\left(2 \pi \alpha^{\prime}\right)} b_{M N} \partial_{\sigma} x^{N}-T_{D 1} C_{M N}^{(2)} \partial_{\sigma} x^{N}
$$

According to the standard treatment of the constraint systems we introduce the extended Hamiltonian with all primary constraints included

$$
H_{E}=\int d \sigma\left(\lambda_{\tau} \mathcal{H}_{\tau}+\lambda_{\sigma} \mathcal{H}_{\sigma}-A_{\tau} \partial_{\sigma} \pi^{\sigma}+v_{\tau} \pi^{\tau}\right),
$$

where $\lambda_{\tau, \sigma}$ and $v_{\tau}$ are Lagrange multipliers corresponding to the constraints $\mathcal{H}_{\tau}, \mathcal{H}_{\sigma}$ and $\pi^{\tau}$. Now the requirement of the preservation of the primary constraint $\pi^{\tau} \approx 0$ implies the secondary constraint

$$
\mathcal{G}=\partial_{\sigma} \pi^{\sigma} \approx 0 .
$$

We should also check that the constraints $\mathcal{H}_{\tau}, \mathcal{H}_{\sigma}$ are preserved during the time evolution as well. Performing the same calculations as in [3] we obtain

$$
\left\{\mathcal{H}_{\tau}(\sigma), \mathcal{H}_{\tau}\left(\sigma^{\prime}\right)\right\} \approx 0, \quad\left\{\mathcal{H}_{\tau}(\sigma), \mathcal{H}_{\sigma}\left(\sigma^{\prime}\right)\right\} \approx 0, \quad\left\{\mathcal{H}_{\sigma}(\sigma), \mathcal{H}_{\sigma}\left(\sigma^{\prime}\right)\right\} \approx 0 .
$$

In other words $\mathcal{H}_{\tau} \approx 0, \mathcal{H}_{\sigma} \approx 0$ and $\mathcal{G} \approx 0$ are the first class constraints. Our goal is to find the formulation of the D1-brane action where these first class constraints are fixed. To be more specific let us presume that the background has the form [4-6]

$$
\begin{aligned}
d s^{2} & =g_{M N} d x^{M} d x^{N}=g_{t t} d t^{2}+g_{\varphi \varphi} d \varphi^{2}+g_{\mu \nu} d x^{\mu} d x^{\nu}, \\
B & =b_{M N} d X^{M} d X^{N}=b_{\mu \nu} d x^{\mu} d x^{\nu}
\end{aligned}
$$


where $\mu, \nu$ denote the transverse directions. Following $[4,5]$ we introduce light cone coordinates

$$
x^{-}=\varphi-t, \quad x^{+}=(1-a) t+a \varphi
$$

with inverse relations

$$
t=x^{+}-a x^{-}, \quad \varphi=x^{+}+(1-a) x^{-} .
$$

Then corresponding metric components have the form

$$
G_{++}=g_{t t}+g_{\varphi \varphi}, \quad G_{--}=g_{t t} a^{2}+(1-a)^{2} g_{\varphi \varphi}, \quad G_{+-}=-a g_{t t}+(1-a) g_{\varphi \varphi}
$$

with inverse

$$
\begin{aligned}
G^{++} & =\frac{g_{t t} a^{2}+(1-a)^{2} g_{\varphi \varphi}}{g_{t t} g_{\varphi \varphi}}, \quad G^{--}=\frac{g_{t t}+g_{\varphi \varphi}}{g_{t t} g_{\varphi \varphi}}, \\
G^{+-} & =\frac{a g_{t t}-(1-a) g_{\varphi \varphi}}{g_{t t} g_{\varphi \varphi}}
\end{aligned}
$$

Further, using the relation between light-cone coordinates and the original ones we obtain following components of Ramond-Ramond two form

$$
\begin{aligned}
& C_{+-}^{(2)}=-C_{-+}^{(2)}=C_{t \varphi}^{(2)}, \\
& C_{+\mu}^{(2)}=-C_{\mu+}=C_{t \mu}^{(2)}+C_{\varphi \mu}^{(2)}, \\
& C_{-\mu}^{(2)}=-C_{\mu-}^{(2)}=-C_{t \mu}^{(2)}+(1-a) C_{\varphi \mu}^{(2)} .
\end{aligned}
$$

In the light cone coordinates the Hamiltonian and diffeomorphism constraints have the form

$$
\begin{aligned}
\mathcal{H}_{\tau}= & \Pi_{+} G^{++} \Pi_{+}+2 \Pi_{+} G^{+-} \Pi_{-}+\Pi_{-} G^{--} \Pi_{-} \\
& +\left(T_{D 1}^{2} e^{-2 \Phi}+\left(\frac{\pi_{\sigma}}{2 \pi \alpha^{\prime}}-T_{D 1} C^{(0)}\right)^{2}\right) \begin{array}{l}
\left(G_{++}\left(\partial_{\sigma} x^{+}\right)^{2}\right. \\
\left.+2 G_{+-} \partial_{\sigma} x^{+} \partial_{\sigma} x^{-}+G_{--}\left(\partial_{\sigma} x^{-}\right)^{2}\right)+\mathcal{H}_{x}
\end{array} \\
\mathcal{H}_{\sigma}= & p_{+} \partial_{\sigma} x^{+}+p_{-} \partial_{\sigma} x^{-}+p_{\mu} \partial_{\sigma} x^{\mu},
\end{aligned}
$$

where

$$
\mathcal{H}_{x} \equiv \Pi_{\mu} g^{\mu \nu} \Pi_{\nu}+T_{D 1}^{2} e^{-2 \Phi} g_{\mu \nu} \partial_{\sigma} x^{\mu} \partial_{\sigma} x^{\nu}+\left(\frac{1}{\left(2 \pi \alpha^{\prime}\right)} \pi^{\sigma}-T_{D 1} C^{(0)}\right)^{2} \partial_{\sigma} x^{\mu} g_{\mu \nu} \partial_{\sigma} x^{\nu} .
$$

Now we are ready to impose the uniform light cone gauge fixing when we introduce two gauge fixing constraints

$$
\mathcal{G}_{+} \equiv x^{+}-\tau \approx 0, \quad \mathcal{G}_{-} \equiv p_{-}-J \approx 0 .
$$

It is now easy to see that these constraints form the second class constraints together with $\mathcal{H}_{\tau}, \mathcal{H}_{\sigma}$. Further, since we are interested in the Hamiltonian for the physical degrees of freedom we also fix the gauge symmetry generated by the constraint $\mathcal{G}=\partial_{\sigma} \pi^{\sigma} \approx 0$. We fix this symmetry by imposing the condition $A_{\sigma}=$ const. Then from the equation of motion 
for $\pi^{\sigma}$ we find that $\pi^{\sigma}=\pi^{\sigma}(\sigma)$. Finally using the strong form of the constraint $\mathcal{G}$ we find that $\pi^{\sigma}=$ const. Then the action has the form

$$
\begin{aligned}
S & =\int d \tau d \sigma\left(p_{+} \partial_{\tau} x^{+}+p_{-} \partial_{\tau} x^{-}+p_{\mu} \partial_{\tau} x^{\mu}-H_{\text {fixed }}\right) \\
& =\int d \tau d \sigma\left(p_{\mu} x^{\mu}+p_{+}\right)
\end{aligned}
$$

where we used the fact that $H_{\text {fixed }}$ is the sum of the second class constraints that vanish strongly. From the previous expression we see that we can identify the Hamiltonian for the reduced system as

$$
\mathcal{H}_{\text {red }}=-p_{+} .
$$

Now using $\mathcal{H}_{\tau}=0, \mathcal{H}_{\sigma}=0$ and $\mathcal{G}_{+}=0, \mathcal{G}_{-}=0$ we obtain

$$
\partial_{\sigma} x^{-}=-\frac{1}{J} p_{\mu} \partial_{\sigma} x^{\mu}
$$

and hence $\mathcal{H}_{\tau}$ is equal to

$$
\begin{aligned}
\mathcal{H}_{\tau}= & \Pi_{+} G^{++} \Pi_{+}+2 \Pi_{+} G^{+-} \Pi_{-}+\Pi_{-} G^{--} \Pi_{-} \\
& +\left(T_{D 1}^{2} e^{-2 \Phi}+\left(\frac{\pi_{\sigma}}{2 \pi \alpha^{\prime}}-T_{D 1} C^{(0)}\right)^{2}\right) G_{--}\left(\partial_{\sigma} x^{-}\right)^{2}+\mathcal{H}_{x}=0 .
\end{aligned}
$$

We solve this equation for $\Pi_{+}$and we obtain

$$
\Pi_{+}=\frac{-2 G^{+-} \Pi_{-}-\sqrt{\mathbf{K}}}{2 G^{++}},
$$

where

$$
\Pi_{+}=p_{+}-T_{D 1} C_{+\mu}^{(2)} \partial_{\sigma} x^{\mu}+\frac{T_{D 1}}{J} C_{+-}^{(2)}\left(p_{\mu} \partial_{\sigma} x^{\mu}\right),
$$

and where

$$
\begin{aligned}
\mathbf{K}= & 4\left(G^{+-} \Pi_{-}\right)^{2} \\
& -4 G^{++}\left[G^{--} \Pi_{-}^{2}+\left(T_{D 1}^{2} e^{-2 \Phi}+\left(\frac{\pi^{\sigma}}{\left(2 \pi \alpha^{\prime}\right)}-T_{D 1} C^{(0)}\right)^{2}\right) G_{--} \frac{1}{J^{2}}\left(p_{\mu} \partial_{\sigma} x^{\mu}\right)^{2}+\mathcal{H}_{x}\right] .
\end{aligned}
$$

In order to simplify resulting expressions we use following notations

$$
\begin{aligned}
\Pi_{\mu} & =p_{\mu}-\frac{\pi^{\sigma}}{\left(2 \pi \alpha^{\prime}\right)} b_{\mu \nu} \partial_{\sigma} x^{\nu}-T_{D 1} C_{\mu \nu}^{(2)} \partial_{\sigma} x^{\nu}+T_{D 1} C_{\mu-}^{(2)} \frac{1}{J}\left(p_{\nu} \partial_{\sigma} x^{\nu}\right) \\
& \equiv \mathbf{A}_{\mu}^{\nu} p_{\nu}-\mathbf{V}_{\mu}, \\
\mathbf{V}_{\mu} & =\frac{\pi^{\sigma}}{\left(2 \pi \alpha^{\prime}\right)} b_{\mu \nu} \partial_{\sigma} x^{\nu}+T_{D 1} C_{\mu \nu}^{(2)} \partial_{\sigma} x^{\nu}, \quad \mathbf{A}_{\mu}{ }^{\nu}=\delta_{\mu}{ }^{\nu}+\frac{T_{D 1}}{J} C_{\mu-}^{(2)} \partial_{\sigma} x^{\nu} . \\
\Pi_{-} & =J-T_{D 1} C_{-\mu}^{(2)} \partial_{\sigma} x^{\mu} .
\end{aligned}
$$


To proceed further note that by definition we have the relation

$$
\partial_{\sigma} x^{\mu} p_{\mu}=\frac{1}{1+\frac{T_{D 1}}{J} \partial_{\sigma} x^{\rho} C_{\rho-}^{(2)}} \partial_{\sigma} x^{\mu} \Pi_{\mu} .
$$

Using these formulas we can write $\mathbf{K}$ as

$$
\mathbf{K}=4\left(G^{+-} G^{+-}-G^{++} G^{--}\right) \Pi_{-}^{2}-4 G^{++} \mathbf{B}^{\mu \nu} \Pi_{\mu} \Pi_{\nu}-4 G^{++} V,
$$

where

$$
\begin{aligned}
A & =\left(T_{D 1}^{2} e^{-2 \Phi}+\left(\frac{\pi^{\sigma}}{\left(2 \pi \alpha^{\prime}\right)}-T_{D 1} C^{(0)}\right)^{2}\right) \frac{G_{--}}{J^{2}\left(1+\frac{T_{D 1}}{J} \partial_{\sigma} x^{\rho} C_{\rho-}^{(2)}\right)^{2}}, \\
\mathbf{B}^{\mu \nu} & =A \partial_{\sigma} x^{\mu} \partial_{\sigma} x^{\nu}+g^{\mu \nu} \\
V & =\left(T_{D 1}^{2} e^{-2 \Phi}+\left(\frac{\pi^{\sigma}}{\left(2 \pi \alpha^{\prime}\right)}-T_{D 1} C^{(0)}\right)^{2}\right) g_{\sigma \sigma}, \quad g_{\sigma \sigma}=g_{\mu \nu} \partial_{\sigma} x^{\mu} \partial_{\sigma} x^{\nu} .
\end{aligned}
$$

Collecting these results we derive the Hamiltonian density for the physical degrees of freedom in the form

$$
\mathcal{H}_{\mathrm{red}}=-p_{+}=-T_{D 1} C_{+\mu} \partial_{\sigma} x^{\mu}+\frac{T_{D 1}}{J\left(1+\frac{T_{D 1}}{J} \partial_{\sigma} x^{\rho} C_{\rho-}^{(2)}\right)} \partial_{\sigma} x^{\mu} \Pi_{\mu}+\frac{G^{+-}}{G^{++}} \Pi_{-}+\frac{1}{2 G^{++}} \sqrt{\mathbf{K}}
$$

Our goal is to find the Lagrangian density for the physical degrees of freedom. To do this we use the equation of motion for $x^{\mu}$ that follow from the Hamiltonian principle

$$
\partial_{\tau} x^{\mu}=\left\{x^{\mu}, H_{\text {red }}\right\}=\frac{T_{D 1} C_{+-}^{(2)}}{J\left(1+\frac{T_{D 1}}{J} \partial_{\sigma} x^{\rho} C_{\rho-}^{(2)}\right)} \partial_{\sigma} x^{\nu} \mathbf{A}_{\nu}^{\mu}-\frac{1}{\sqrt{\mathbf{K}}} 2 \Pi_{\rho} \mathbf{B}^{\rho \nu} \mathbf{A}_{\nu}{ }^{\mu} .
$$

We can invert this equation and we obtain

$$
\Pi_{\mu}=-\frac{\sqrt{\mathbf{K}}}{2} \mathbf{X}^{\nu}\left(\mathbf{A}^{-1}\right)_{\nu}{ }^{\rho} \mathbf{B}_{\rho \mu}^{-1}
$$

where

$$
\begin{aligned}
\mathbf{X}^{\mu} & =\partial_{\tau} x^{\mu}-\frac{T_{D 1} C_{+-}^{(2)}}{J\left(1+\frac{T_{D 1}}{J} \partial_{\sigma} x^{\rho} C_{\rho-}^{(2)}\right)} \partial_{\sigma} x^{\nu} \mathbf{A}_{\nu}^{\mu}, \\
\mathbf{B}_{\mu \nu}^{-1} & =g_{\mu \nu}-A \frac{g_{\mu \sigma} \partial_{\sigma} x^{\sigma} \partial_{\sigma} x^{\omega} g_{\omega \nu}}{1+A g_{\sigma \sigma}}, \\
\left(\mathbf{A}^{-1}\right)_{\mu}^{\nu} & =\delta_{\mu}{ }^{\nu}-\frac{T_{D 1}}{J} \frac{C_{\mu-} \partial_{\sigma} x^{\nu}}{1+\frac{T_{D 1}}{J} \partial_{\sigma} x^{\mu} C_{\mu-}} .
\end{aligned}
$$

Using (2.34) we obtain the Lagrangian density for the physical degrees of freedom

$$
\begin{aligned}
\mathcal{L}_{\text {red }}= & p_{\mu} \partial_{\tau} x^{\mu}-\mathcal{H}_{\text {red }} \\
= & \left(\mathbf{X}^{\nu}+\frac{T_{D 1} C_{+-}^{(2)}}{J\left(1+\frac{T_{D 1}}{J} \partial_{\sigma} x^{\rho} C_{\rho-}^{(2)}\right)} \partial_{\sigma} x^{\sigma} \mathbf{A}_{\sigma}{ }^{\nu}\right)\left(\mathbf{A}^{-1}\right)_{\nu}{ }^{\mu} \mathbf{V}_{\mu} \\
& +T_{D 1} C_{+\mu} \partial_{\sigma} x^{\mu}-\frac{G^{+-}}{G^{++}} \Pi_{-}+\frac{2}{G^{++} \sqrt{\mathbf{K}}}\left(G^{++} V-\left(G^{+-} G^{+-}-G^{++} G^{--}\right)\right) .
\end{aligned}
$$


However given Lagrangian still depends on conjugate momenta $p_{\mu}$ through the function $\mathbf{K}$. In order to express $\mathbf{K}$ as the function of time derivatives of $x^{\mu}$ we insert (2.33) into the definition of $\mathbf{K}(2.29)$ and we obtain

$$
\mathbf{K}=\frac{4}{1+G^{++} \mathbf{X}^{\rho}\left(\mathbf{A}^{-1}\right)_{\rho}^{\mu} \mathbf{B}_{\mu \nu} \mathbf{X}^{\omega}\left(\mathbf{A}^{-1}\right)_{\omega}^{\nu}}\left(\left(G^{+-} G^{+-}-G^{++} G^{--}\right) \Pi_{-}^{2}-G^{++} V\right)
$$

so that the Lagrangian density $\mathcal{L}_{\text {red }}$ has the form

$$
\begin{aligned}
\mathcal{L}_{\mathrm{red}}= & \left(\mathbf{X}^{\mu}+\frac{T_{D 1} C_{+-}^{(2)}}{J\left(1+\frac{T_{D 1}}{J} \partial_{\sigma} x^{\rho} C_{\rho-}^{(2)}\right)} \partial_{\sigma} x^{\nu} \mathbf{A}_{\nu}^{\mu}\right)\left(\mathbf{A}^{-1}\right)_{\nu}^{\mu} \mathbf{V}_{\mu} \\
& +T_{D 1} C_{+\mu} \partial_{\sigma} x^{\mu}-\frac{G^{+-}}{G^{++}} \Pi_{-} \\
& -\frac{1}{G^{++}} \sqrt{-\left(1+G^{++} \mathbf{X}^{\rho}\left(\mathbf{A}^{-1}\right)_{\rho}^{\mu} \mathbf{B}_{\mu \nu} \mathbf{X}^{\omega}\left(\mathbf{A}^{-1}\right)_{\omega}^{\nu}\right)\left(G^{++} V-\left(G^{+-} G^{+-}-G^{++} G^{--}\right)\right)} .
\end{aligned}
$$

We simplify given expression using

$$
\left(\mathbf{X}^{\mu}+\frac{T_{D 1} C_{+-}^{(2)}}{J\left(1+\frac{T_{D 1}}{J} \partial_{\sigma} x^{\rho} C_{\rho-}^{(2)}\right)} \partial_{\sigma} x^{\nu} \mathbf{A}_{\nu}^{\mu}\right)\left(\mathbf{A}^{-1}\right)_{\nu}^{\mu} \mathbf{V}_{\mu}=\frac{\pi^{\sigma}}{2 \pi \alpha^{\prime}} \partial_{\tau} x^{\mu} b_{\mu \nu} \partial_{\sigma} x^{\nu}+T_{D 1} \partial_{\tau} x^{\mu} C_{\mu \nu}^{(2)} \partial_{\sigma} x^{\nu}
$$

together with

$$
\begin{aligned}
\left(\mathbf{X A}^{-1}\right)^{\mu} \mathbf{B}_{\mu \nu}^{-1}\left(\mathbf{X A}^{-1}\right)^{\nu}= & \frac{1}{1+A g_{\sigma \sigma}} \\
\times & {\left[1+A g_{\sigma \sigma}+G^{++} g_{\tau \tau}+A G^{++}\left(g_{\tau \tau} g_{\sigma \sigma}-g_{\tau \sigma} g_{\tau \sigma}\right)\right.} \\
& -2 g_{\tau \sigma} G^{++} \frac{T_{D 1}}{J\left(1+\frac{T_{D 1}}{J} \partial_{\sigma} x^{\mu} C_{\mu-}^{(2)}\right)}\left(\partial_{\tau} x^{\mu} C_{\mu-}^{(2)}+C_{+-}\right) \\
& \left.+g_{\sigma \sigma} G^{++}\left(\frac{T_{D 1}}{J\left(1+\frac{T_{D 1}}{J} \partial_{\sigma} x^{\mu} C_{\mu-}^{(2)}\right)}\left(\partial_{\tau} x^{\mu} C_{\mu-}^{(2)}+C_{+-}\right)\right)^{2}\right]
\end{aligned}
$$

Finally using the fact that we can write

$$
G^{++} V+\left(G^{++} G^{--}-G^{+-} G^{+-}\right) \Pi_{-}^{2}=\left(G^{++} G^{--}-G^{+-} G^{+-}\right) \Pi_{-}^{2}\left(1+A g_{\sigma \sigma}\right)
$$

we obtain the light-cone gauge fixed Lagrangian density for D1-brane in the form

$$
\begin{aligned}
\mathcal{L}_{\mathrm{red}}= & \frac{\pi^{\sigma}}{2 \pi \alpha^{\prime}} \partial_{\tau} x^{\mu} b_{\mu \nu} \partial_{\sigma} x^{\nu}+T_{D 1} \partial_{\tau} x^{\mu} C_{\mu \nu}^{(2)} \partial_{\sigma} x^{\nu}+T_{D 1} C_{+\mu}^{(2)} \partial_{\sigma} x^{\mu} \\
& -\frac{G^{+-}}{G^{++}}\left(J+T_{D 1} C_{\mu-}^{(2)} \partial_{\sigma} x^{\mu}\right)-\sqrt{-\frac{1}{G^{++} G_{--}}\left(J+T_{D 1} C_{\mu-}^{(2)} \partial_{\sigma} x^{\mu}\right)^{2} \mathcal{K}}
\end{aligned}
$$


where

$$
\begin{aligned}
\mathcal{K}= & 1+\left(e^{-2 \Phi}+\left(\pi^{\sigma}-C^{(0)}\right)^{2}\right) \frac{T_{D 1}^{2}}{J^{2}} \frac{G_{--}}{\left(1+\frac{T_{D 1}}{J} \partial_{\sigma} x^{\rho} C_{\rho-}^{(2)}\right)^{2}} g_{\sigma \sigma} \\
& +G^{++} g_{\tau \tau}+\left(e^{-2 \Phi}+\left(\pi^{\sigma}-C^{(0)}\right)^{2}\right) \frac{T_{D 1}^{2}}{J^{2}} \frac{G^{++} G_{--}}{\left(1+\frac{T_{D 1}}{J} \partial_{\sigma} x^{\rho} C_{\rho-}^{(2)}\right)^{2}}\left(g_{\tau \tau} g_{\sigma \sigma}-g_{\tau \sigma} g_{\tau \sigma}\right) \\
& -2 \frac{T_{D 1}}{J} g_{\tau \sigma} G^{++} \frac{1}{1+\frac{T_{D 1}}{J} \partial_{\sigma} x^{\mu} C_{\mu-}^{(2)}}\left(\partial_{\tau} x^{\mu} C_{\mu-}^{(2)}+C_{+-}^{(2)}\right) \\
& +\frac{T_{D 1}^{2}}{J^{2}} g_{\sigma \sigma} G^{++}\left(\frac{1}{1+\frac{T_{D 1}}{J} \partial_{\sigma} x^{\mu} C_{\mu-}^{(2)}}\left(\partial_{\tau} x^{\mu} C_{\mu-}^{(2)}+C_{+-}^{(2)}\right)\right)^{2},
\end{aligned}
$$

and where we also used the fact that $T_{D 1}=\frac{1}{2 \pi \alpha^{\prime}}$. If we now perform rescaling the spatial coordinate as

$$
\frac{J}{T_{D 1}} \sigma=\sigma^{\prime}
$$

we obtain the action in the form

$$
S=\int d \sigma^{\prime} d \tau \mathcal{L}_{\text {red }}
$$

where

$$
\begin{aligned}
\mathcal{L}_{\mathrm{red}}= & \frac{\pi^{\sigma}}{2 \pi \alpha^{\prime}} \partial_{\tau} x^{\mu} b_{\mu \nu} \partial_{\sigma^{\prime}} x^{\nu}+T_{D 1} \partial_{\tau} x^{\mu} C_{\mu \nu}^{(2)} \partial_{\sigma^{\prime}} x^{\nu}+T_{D 1} C_{+\mu}^{(2)} \partial_{\sigma^{\prime}} x^{\mu} \\
& -T_{D 1} \frac{G^{+-}}{G^{++}}\left(1+C_{\mu-}^{(2)} \partial_{\sigma^{\prime}} x^{\mu}\right)-T_{D 1} \sqrt{-\frac{1}{G^{++} G_{--}}\left(1+C_{\mu-}^{(2)} \partial_{\sigma^{\prime}} x^{\mu}\right)^{2} \mathcal{K}} \\
\mathcal{K}= & 1+\left(e^{-2 \Phi}+\left(\pi^{\sigma}-C^{(0)}\right)^{2}\right) \frac{G_{--}}{\left(1+\partial_{\sigma^{\prime}} x^{\rho} C_{\rho-}^{(2)}\right)^{2}} g_{\sigma \sigma} \\
& +G^{++} g_{\tau \tau}+\left(e^{-2 \Phi}+\left(\pi^{\sigma}-C^{(0)}\right)^{2}\right) \frac{G^{++} G_{--}}{\left(1+\partial_{\sigma^{\prime}} x^{\rho} C_{\rho-}^{(2)}\right)^{2}}\left(g_{\tau \tau} g_{\sigma \sigma}-g_{\tau \sigma} g_{\tau \sigma}\right) \\
& -2 g_{\tau \sigma} G^{++} \frac{1}{1+\partial_{\sigma^{\prime}} x^{\mu} C_{\mu-}^{(2)}}\left(\partial_{\tau} x^{\mu} C_{\mu-}^{(2)}+C_{+-}^{(2)}\right) \\
& +g_{\sigma \sigma} G^{++}\left(\frac{1}{1+\partial_{\sigma^{\prime}} x^{\mu} C_{\mu-}^{(2)}}\left(\partial_{\tau} x^{\mu} C_{\mu-}^{(2)}+C_{+-}^{(2)}\right)\right)^{2} .
\end{aligned}
$$

This is the final form of gauge fixed D1-brane in general background. It is easy to see that when Ramond-Ramond fields vanish, $\Phi=$ const and when $\pi^{\sigma}=0$ the action has formally the same form as the gauge-fixed form of the fundamental string action in arbitrary background $g_{M N}, b_{M N}$ with vanishing light-cone components of $B$ that was derived in [5]. Of course, strictly speaking these two actions coincide in the limit of infinite long string since we started with D1-brane that is infinite extended. 


\section{Double Wick rotation}

In this section we perform double Wick rotation in case of the gauge fixed Lagrangian density given in (2.45) following very nice analysis performed in [6] where also very interesting conjecture related to the double Wick rotation was suggested. It was argued there that due to the fact that the light cone gauge fixed $\mathrm{AdS}_{5} \times S^{5}$ string is not Lorentz invariant we obtain that the double Wick rotation gives an inequivalent theory, so called mirror theory. Since this mirror symmetry is important in the study of the integrability of the string in $\mathrm{AdS}_{5} \times S^{5}$ it was suggested in [6] that the double Wick rotation could be fundamental in the definition of the mirror theory. Explicitly, it was shown in [6] that the application of the double Wick rotation to the case of the light cone gauge fixed string on some background gives the string theory formulated on another background.

Due to the similarity between fundamental string action and D1-brane action we would like to extend given idea to the case of the light-cone gauge fixed D1-brane action introduced above. Clearly given action is more complicated due to the explicit coupling of D1-brane to the dilaton and Ramond-Ramond fields which is invisible in the classical bosonic string. For that reason it is instructive to see how the action (2.45) changes under double Wick rotation that is defined as

$$
\tau=i \tilde{\sigma}, \quad \sigma^{\prime}=-i \tilde{\tau}
$$

so that

$$
\frac{\partial}{\partial \tau}=-i \frac{\partial}{\partial \tilde{\sigma}}, \quad \frac{\partial}{\partial \sigma^{\prime}}=i \frac{\partial}{\partial \tilde{\tau}}
$$

Now under these transformations $\mathcal{K}$ takes the form

$$
\begin{aligned}
\mathcal{K}= & 1-\left(e^{-2 \Phi}+\left(\pi^{\sigma}-C^{(0)}\right)^{2}\right) \frac{G_{--}}{\left(1+i \partial_{\tilde{\sigma}} x^{\rho} C_{\rho-}^{(2)}\right)^{2}} g_{\tilde{\tau} \tilde{\tau}} \\
& -G^{++} g_{\tilde{\sigma} \tilde{\sigma}}+\left(e^{-2 \Phi}+\left(\pi^{\sigma}-C^{(0)}\right)^{2}\right) \frac{G^{++} G_{--}}{\left(1+i \partial_{\left.\tilde{\tau} x^{\rho} C_{\rho-}^{(2)}\right)^{2}}\right.}\left(g_{\tilde{\tau} \tilde{\tau}} g_{\tilde{\sigma} \tilde{\sigma}}-g_{\tilde{\tau} \tilde{\sigma}} g_{\tilde{\tau} \tilde{\sigma}}\right) \\
& -2 g_{\tilde{\tau} \tilde{\sigma} G^{++}} \frac{1}{\left(1+i \partial_{\tilde{\tau}} x^{\mu} C_{\mu-}^{(2)}\right)}\left(-i \partial_{\tilde{\tau}} x^{\mu} C_{\mu-}^{(2)}+C_{+-}\right) \\
& -g_{\tilde{\tau} \tilde{\tau}} G^{++}\left(\frac{1}{\left(1+i \partial_{\tilde{\tau}} x^{\mu} C_{\mu-}^{(2)}\right)}\left(-\partial_{\tilde{\sigma}} x^{\mu} C_{\mu-}^{(2)}+C_{+-}\right)\right)^{2}
\end{aligned}
$$

We see that in order to have the same form of the Lagrangian as the original one we should demand that

$$
C_{\rho-}^{(2)}=0
$$

and we return to this important restriction letter. Let us denote the background fields in the mirror theory with tilde. Then in order to have mirror theory in the same form as the original one we obtain following relations between mirror background fields and 
original ones

$$
\begin{aligned}
e^{-2 \Phi}+\left(\pi^{\sigma}-C^{(0)}\right)^{2} & =e^{-2 \tilde{\Phi}}+\left(\pi^{\sigma}-\tilde{C}^{(0)}\right)^{2}, \\
\tilde{G}^{++} & =-\left(e^{-2 \Phi}+\left(\pi^{\sigma}-C^{(0)}\right)^{2}\right) G_{--}-G^{++} C_{+-}^{2}, \\
\tilde{G}_{--} & =-\frac{G^{++} G_{--}}{\left(e^{-2 \Phi}+\left(\pi^{\sigma}-C^{(0)}\right)^{2}\right) G_{--}+G^{++} C_{+-}^{2}}, \\
\tilde{C}_{+-} & =-\frac{G^{++} C_{+-}}{\left(e^{-2 \Phi}+\left(\pi^{\sigma}-C^{(0)}\right)^{2}\right) G_{--}+G^{++} C_{+-}^{2}} \\
\tilde{G}^{+-} & =-\frac{G^{+-}}{G^{++}}\left(e^{-2 \Phi}+\left(\pi^{\sigma}-C^{(0)}\right)^{2}\right) G_{--}-G^{+-} C_{+-}^{2}, \\
b_{\mu \nu} & =-\tilde{b}_{\mu \nu}, \quad C_{\mu \nu}^{(2)}=-\tilde{C}_{\mu \nu}^{(2)} .
\end{aligned}
$$

Let us now comments given results. The most important one is on the first line that shows that we are not able to determine the transformation properties of $\Phi$ and $C^{(0)}$ separately while their combination that includes the world-volume electric flux is constant. This is the first issue since the double Wick rotation seems to mix background fields with the worldvolume ones. Secondly, even in the case of the simplest background $\mathrm{AdS}_{5} \times S^{5}$ this result seems to predict different transformations of dilaton from the result determined in [6]. Of course, there is still a possibility that non-trivial dilaton dependence in the mirror theory is compensated by non-trivial behavior of $C^{(0)}$ so that their combination is constant but it seems that such configurations do not solve the supergravity equations of motion. Most importantly we see from (3.4) that the mirror theory has the same form as the original one when we have to impose the restriction on the background that $C_{-\mu}^{(2)}=0$. However it is easy to see that this condition certainly cannot be obeyed in case of $\kappa$-deformed $\mathrm{AdS}_{3} \times S^{3}$ background that was found in [16]. We also mean that this condition $C_{-\mu}^{(2)}=0$ cannot be imposed as the restriction on the possible form of the background since DBI action is the effective description of D-brane dynamics and there is no fundamental principle that would say that uniform gauge fixed D1-brane theory had to possess mirror symmetry. For these reasons we mean that the double Wick rotation in case of gauge fixed D1-brane action generally gives theory with different Lagrangian density so that it does not make sense to speak about Wick rotated D1-brane theory as mirror theory.

It is possible that the reason why D1-brane theory behaves differently from the fundamental string action is in the fact that the double Wick rotation performed in case of the fundamental string corresponds to the T-duality in $\phi$ coordinate, then performing analytic continuing $t, \phi \rightarrow i \phi,-i t$ and doing another $T$-duality in the new $\phi$ coordinate [15]. On the other hand it is not known how to perform such a T-duality transformations in case of the uniform gauge fixed D1-brane action however it is possible that such an operation is not equivalent to the double Wick rotation performed above. We hope to return to this problem in future. 


\section{Acknowledgments}

This work was supported by the Grant agency of the Czech Republic under the grant P201/12/G028.

Open Access. This article is distributed under the terms of the Creative Commons Attribution License (CC-BY 4.0), which permits any use, distribution and reproduction in any medium, provided the original author(s) and source are credited.

\section{References}

[1] G. Arutyunov and S. Frolov, Foundations of the $A d S_{5} \times S^{5}$ superstring: I, J. Phys. A 42 (2009) 254003 [arXiv:0901.4937] [inSPIRE].

[2] N. Beisert et al., Review of AdS/CFT integrability: an overview, Lett. Math. Phys. 99 (2012) 3 [arXiv: 1012.3982] [INSPIRE].

[3] J. Klusoň, Integrability of D1-brane on group manifold, JHEP 09 (2014) 159 [arXiv:1407.7665] [INSPIRE].

[4] G. Arutyunov, R. Borsato and S. Frolov, S-matrix for strings on $\eta$-deformed $A d S_{5} \times S^{5}$, JHEP 04 (2014) 002 [arXiv: 1312.3542] [INSPIRE].

[5] G. Arutyunov, M. de Leeuw and S.J. van Tongeren, The exact spectrum and mirror duality of the $\left(A d S_{5} \times S^{5}\right)_{\eta}$ superstring, Theor. Math. Phys. 182 (2015) 23

[Teor. Mat. Fiz. 182 (2014) 28] [arXiv:1403.6104] [INSPIRE].

[6] G. Arutyunov and S.J. van Tongeren, $A d S_{5} \times S^{5}$ mirror model as a string $\sigma$-model, Phys. Rev. Lett. 113 (2014) 261605 [arXiv:1406.2304] [INSPIRE].

[7] G. Arutyunov and S. Frolov, On string S-matrix, bound states and TBA, JHEP 12 (2007) 024 [arXiv:0710.1568] [inSPIRE].

[8] J. Polchinski, Dirichlet branes and Ramond-Ramond charges, Phys. Rev. Lett. 75 (1995) 4724 [hep-th/9510017] [INSPIRE].

[9] G. Arutyunov and S. Frolov, String hypothesis for the $A d S_{5} \times S^{5}$ mirror, JHEP 03 (2009) 152 [arXiv:0901.1417] [INSPIRE].

[10] G. Arutyunov and S. Frolov, Thermodynamic Bethe Ansatz for the $A d S_{5} \times S^{5}$ mirror model, JHEP 05 (2009) 068 [arXiv:0903.0141] [INSPIRE].

[11] B. Basso, A. Sever and P. Vieira, Spacetime and flux tube S-matrices at finite coupling for $\mathcal{N}=4$ supersymmetric Yang-Mills theory, Phys. Rev. Lett. 111 (2013) 091602 [arXiv: 1303.1396] [INSPIRE].

[12] B. Basso, A. Sever and P. Vieira, Space-time S-matrix and flux tube S-matrix II. Extracting and matching data, JHEP 01 (2014) 008 [arXiv:1306.2058] [INSPIRE].

[13] B. Basso, A. Sever and P. Vieira, Space-time S-matrix and flux-tube S-matrix III. The two-particle contributions, JHEP 08 (2014) 085 [arXiv: 1402.3307] [INSPIRE].

[14] F. Delduc, M. Magro and B. Vicedo, An integrable deformation of the $A d S_{5} \times S^{5}$ superstring action, Phys. Rev. Lett. 112 (2014) 051601 [arXiv: 1309.5850] [INSPIRE].

[15] G. Arutyunov and S.J. van Tongeren, Double Wick rotating Green-Schwarz strings, JHEP 05 (2015) 027 [arXiv: 1412.5137] [INSPIRE]. 
[16] O. Lunin, R. Roiban and A.A. Tseytlin, Supergravity backgrounds for deformations of $A d S_{n} \times S^{n}$ supercoset string models, Nucl. Phys. B 891 (2015) 106 [arXiv:1411.1066] [INSPIRE].

[17] F. Delduc, M. Magro and B. Vicedo, Derivation of the action and symmetries of the q-deformed $A d S_{5} \times S^{5}$ superstring, JHEP 10 (2014) 132 [arXiv:1406.6286] [INSPIRE].

[18] B. Hoare, R. Roiban and A.A. Tseytlin, On deformations of $A d S_{n} \times S^{n}$ supercosets, JHEP 06 (2014) 002 [arXiv: 1403.5517] [INSPIRE].

[19] S.J. van Tongeren, Integrability of the $A d S_{5} \times S^{5}$ superstring and its deformations, J. Phys. A 47 (2014) 433001 [arXiv:1310.4854] [INSPIRE].

[20] J. Simon, Brane effective actions, $\kappa$-symmetry and applications, Living Rev. Rel. 15 (2012) 3 [arXiv: 1110.2422] [INSPIRE].

[21] M. Kruczenski, A.V. Ryzhov and A.A. Tseytlin, Large spin limit of $A d S_{5} \times S^{5}$ string theory and low-energy expansion of ferromagnetic spin chains, Nucl. Phys. B 692 (2004) 3 [hep-th/0403120] [INSPIRE].

[22] G. Arutyunov and S. Frolov, Integrable Hamiltonian for classical strings on $A d S_{5} \times S^{5}$, JHEP 02 (2005) 059 [hep-th/0411089] [INSPIRE]. 